\title{
Determination of Phthalate Plasticisers in Palm Oil Using Online Solid Phase Extraction-Liquid Chromatography (SPE-LC)
}

\author{
Nazarudin Ibrahim, ${ }^{1,2}$ Rozita Osman, ${ }^{2}$ Azmui Abdullah, ${ }^{2}$ and Norashikin Saim ${ }^{2}$ \\ ${ }^{1}$ Chemistry Department of Malaysia, Jalan Sultan, 46661 Petaling Jaya, Selangor, Malaysia \\ ${ }^{2}$ Faculty of Applied Sciences, Universiti Teknologi MARA, 40450 Shah Alam, Selangor, Malaysia \\ Correspondence should be addressed to Norashikin Saim; noras691@salam.uitm.edu.my
}

Received 7 October 2013; Accepted 2 January 2014; Published 19 February 2014

Academic Editor: Cato Brede

Copyright (C) 2014 Nazarudin Ibrahim et al. This is an open access article distributed under the Creative Commons Attribution License, which permits unrestricted use, distribution, and reproduction in any medium, provided the original work is properly cited.

Contamination of phthalates plasticisers to food has raised concern as some of the phthalates are suspected to be endocrine disruptors. The phthalates have high affinity with oily environment and analysing these chemicals in such matrices is difficult because of the trace amount of the analyte and interference from matrix. An online solid phase extraction (SPE) technique using a large volume $(3.5 \mathrm{~mL})$ injection was developed for the analysis of 6 common plasticisers in palm oil. A simple sample preparation involving alumina as a fat retainer and methanol : acetonitrile $(1: 1)$ as the extraction solvent was performed prior to the usage of online SPE-LC system. This system consists of two columns, $\mathrm{C}_{16}$ for the solid phase extraction (SPE) and $\mathrm{C}_{18}$ as the analytical column, and a photo diode array detector. The calibration curves were linear from 5 to $1000 \mu \mathrm{g} \mathrm{L}^{-1}$, with correlation coefficients above 0.99 . The instrumental limit of detection was $3 \mu \mathrm{g} \mathrm{L}^{-1}$ and satisfactory recovery was obtained. A screening on a few samples in the retail market revealed the presence of dibutyl phthalate (DBP) and butylbenzylphthalate (BBP) in the palm oil, with concentration less than $1 \mathrm{mg} \mathrm{L}^{-1}$.

\section{Introduction}

Food packaging is one of the most essential elements in the whole distribution chain of food production. Food packaging minimises product deterioration from oxidation, loss of gas, moisture, UV radiation, foreign aroma influences, temperature instability, microbiological activities, extends shell life, and maintains the quality and safety of the food [1]. Over the past few decades, plastic has replaced metals and glass in many food containers. Plastic possesses advantages over other packaging materials as they can be made into sheets, shapes, and structures, offering limitless design of flexibility. They are chemical resistant which is inexpensive and possess wide range of physical and optical properties and offer a wide angle of product design and function [2]. Most plastic polymers contain many additives, such as antioxidants, plasticisers, and colorants, which are necessary for processing and improving the quality of the final product in terms of durability, flexibility, and appearances. The additives in plastic could be in the form of antioxidant, stabilizers, lubricants, and antistatic and antiblocking agents [3]. The addition of plasticisers, for example, increases the flexibility and workability of some rigid polymers [4]. These plasticisers are low molecular weight substances which are not chemically bonded to long polymer chains and could potentially migrate to food [5-7]. Phthalic acid diesters, for example, are the most common additives and are produced all over the world in large quantities [8]. Humans exposed to these phthalates through ingestion, when the phthalates were used as plasticisers in food packaging [9]. The migration of theses phthalates from food packaging into fatty food matrices is a well-known source of food contamination [10]. The presence of phthalate in food raises concerns because recent studies suggest that certain phthalates can disrupt the endocrine system [11]. Male reproduction system can be adversely affected by dibutyl phthalates, benzyl butyl phthalate, and diethylhexyl phthalate by damaging the deoxyribonucleic acid (DNA) in sperm [12]. The European Union (EU), under the Frame Regulation (EC) 
Number 1935/2004 [13], has mandated that there should be no transfer of constituents of materials or articles intended to come in contact with food in quantities that could either endanger human health or bring about unacceptable changes in the composition or characteristics of the food stuffs.

An analysis of phthalates in oil involves two primary difficulties: firstly, the extraction and separation of the plasticiser from the bulk lipids and secondly from the low concentration of phthalate naturally present in food matrices [14]. The analysis of additives such as phthalates in oil and fat has been studied in several papers. Direct injection of olive oil containing antioxidants into an HPLC was proposed by Quinto-Fernández et al. [15]. To achieve the necessary sensitivity, several methods in analysing phthalates in oil environments involving sample preconcentrations have been proposed. The determination of 6 phthalates in vegetable oil using headspace solid phase microextraction (SPME) paired with gas chromatography employing an electron capture detector (GC/ECD) after optimizing the solvent demonstrated that methanol facilitates the transfer of all the studied phthalates from the oil matrix to the headspace [16]. In another study, the interference from fats in ham was successfully removed using alumina for the determination of 6 phthalates prior to preconcentration by offline SPE and followed by GC/MSD [17].

The application of a sample preconcentration during the determination of phthalates in other food matrices and plastic containers has also been reported. The determination of 6 phthalates in wine using a headspace technique was achieved by optimising the type of fibre, concentration of sodium chloride, and the extraction temperature [18]. The SPME conditions were optimised for the determination of 8 phthalates in blood bags, transfusion tubing, food packaging bags, and mineral water using a GC/FID analyser [19]. Microdialysis was investigated as an enrichment tool for online HPLC to develop an ecofriendly pretreatment process for determining phthalate esters released from plastics contacted by food into water [20].

Online SPE is a combination of SPE and HPLC whereby the sample enrichment, impurity washing, and analyte elution process from SPE directly into the HPLC analytical column for separation and for both qualitative and quantitative analyses. Online SPE has been successfully used in determining polyaromatic hydrocarbons (PAHs) in water [21], paraquat in water [22], and vitamin B1 in beverages [23].

The consumption of edible oil in the world is increasing rapidly every year. Palm oil is currently the most consumed edible oil in the world with $31.3 \%$ of the total 179.38 million tonnes of world production of oil and fats in year 2011 [24]. The supply and demand for palm oil are dominated by the Asian countries.The palm oil products especially refined oil are usually packed in either hard plastic bottle or soft plastic bag. The interaction of the plastic material from the containers with the palm oil could result in the contamination of the palm oil.

The aim of this study was to develop an analytical method for determining the following six phthalate plasticisers in palm oil using online SPE-HPLC/DAD: diphenyl phthalate $(\mathrm{DPhP})$, dimethyl pthalate (DMP), diethyl phthalate (DEP), dibutylphthalate (DBP), butylbenzylphathalate (BBP), and di-n-propyl phthalate (DnPP). To the best of our knowledge, there are no published results on the application of an online SPE-HPLC to analyse plasticisers in palm oil. Matrix cleanup using different types of alumina and the effect of n-hexane as the diluent were also studied to obtain an efficient analysis.

\section{Experimental}

2.1. Chemicals and Reagents. Deionised water was obtained using an EASYpure II ultrapure water system (Thermo Scientific, IA, USA). HPLC-grade methanol and acetonitrile were purchased from Merck (Darmstadt, Germany). Methanesulphonic acid (MSA) was purchased from Merck (Hohenbrunn, Germany). Ethyl acetate and n-hexane (AR Grade) were purchased from Riendemann Schmidt. All standards of phthalates plasticizers: diphenyl phthalate (DPhP), dimethylpthalate (DMP), diethylphthalate (DEP), dibutylphthalate (DBP), butylbenzylphathalate (BBP), and di-n-propyl phthalate (DnPP) standards, were of high purity (>98\%), and they were purchased from Sigma-Aldrich (St. Louis, MO, USA). Aluminium oxide was purchased from SigmaAldrich (St. Louis, MO, USA); aluminium oxide 90 active neutral and Florisil were purchased from Merck (Darmstadt, Germany). Deionized water was from EASYpure II ultrapure water system.

2.2. Preparation of Phthalate Standard Solutions. Both primary and intermediate stock solutions of the mixed phthalate standards were prepared in ethyl acetate $\left(1000 \mathrm{mg} \mathrm{L}^{-1}\right.$ and $100 \mathrm{mg} \mathrm{L}^{-1}$, resp.). A series of working standards was prepared in water, with concentrations ranging from 5 to $1000 \mu \mathrm{g} \mathrm{L}^{-1}$.

2.3. Preparation of Spiked Sample. The cooking oil samples, packed in a polyethylene bottle, were purchased from the local retail supermarket. Two spiked samples containing 1 and $5 \mathrm{mg} \mathrm{L}^{-1}$ of mixed phthalates were prepared by adding $0.1 \mathrm{~mL}$ and $0.5 \mathrm{~mL}$ of the primary stock solution, respectively, to $100 \mathrm{~mL}$ volumetric flask and making up to volume with palm oil.

2.4. Sample Preparation. Refined cooking palm oil samples, packed in a polyethylene bottle and produced by a local company, were purchased from a retail market. The palm oil $(1 \mathrm{~mL})$ was pipetted into a $17 \mathrm{~mL}$ centrifuge tube, diluted with $0.8 \mathrm{~mL}$ of $\mathrm{n}$-hexane, and mixed thoroughly. Alumina $(2.5 \mathrm{~g})$ was added, and the mixture was kept in vortex for $5 \mathrm{~min}$ before removing the $\mathrm{n}$-hexane under a stream of nitrogen at $45^{\circ} \mathrm{C}$. The plasticiser extraction was accomplished by adding $3 \mathrm{~mL}$ of a methanol: acetonitrile mixture $(1: 1)$ and keeping in vortex for 3 minutes, sonicating for 10 minutes, and centrifuging at 50 hertz for 3 minutes before transferring the supernatant to a $10 \mathrm{~mL}$ volumetric flask. The residue was extracted twice using the same procedure, and the supernatants were combined; the volume was reduced to $1 \mathrm{~mL}$ and reconstituted with water. Finally, the solution was 
manually injected into the large loop of the SPE-LC system using syringe.

2.5. Instrumentation and Chromatographic Conditions. The system consists of an online SPE coupled with a DionexUltiMate 3000 x2 Dual HPLC pumping system, automatic injector, two valves of six ports each, a large-volume injection loop $(3.5 \mathrm{~mL})$, and a photodiode array detector. The SPE column was an Acclaim Polar Advantage with the following features: C16, $5 \mu \mathrm{m} 120 \AA$, and $4.6 \times 50 \mathrm{~mm}$. The analytical column was an Acclaim Polar Advantage II with the following features: C18, $5 \mu \mathrm{m} 120 \AA$, and $4.6 \times 150 \mathrm{~mm}$. Both columns were purchased from Dionex (Sunnyvale, CA, USA). The temperature for both the extraction and separation columns was set to $40^{\circ} \mathrm{C}$. The wavelength range used by the photodiode array detector to detect the phthalates was $214-282 \mathrm{~nm}$. The solvent system used was a mixture of ultrapure water and $10 \mathrm{mM}$ methanesulphonic acid (MSA), acetonitrile (ACN), and ethyl acetate. The ports position for each valve and the valve-column switching are shown in Figures 1 and 2, respectively, and the chromatographic conditions for both the SPE and HPLC are shown with valve switching in Table 1. The flow rate was fixed at $1 \mathrm{~mL}$ per minute for all solvent composition.

\section{Results and Discussion}

3.1. Online SPE-HPLC Method Development. The sorption capacity of the SPE column for the online SPE was investigated by testing it with different concentrations of the standards with a fixed loading volume from the large loop [25]. The SPE column was connected to the left pump (injection pump), and the outlet was connected directly to the detector. Two mixed standard solutions of the phthalates (20 and $50 \mathrm{mg} \mathrm{L}^{-1}$ ) were prepared by pipetting 2 and $5 \mathrm{~mL}$ of the primary stock solution, respectively, into $100 \mathrm{~mL}$ volumetric flask, premixed with few drops of methanol, and making up to volume with deionised water. This mixture was then manually loaded onto the large volume loops, with the capacity of approximately $3.5 \mathrm{~mL}$, using a syringe and by means of negative pressure. After the initial valve switching, the sample from the large volume loop was loaded into the SPE column using a mobile phase containing $95 \% 10 \mathrm{mM}$ MSA and 5\% acetonitrile over 15 minutes. During sample loading, all of the analytes were successfully trapped by the SPE column, which was shown by the lack of anelution peak during the first 15 minutes of the chromatogram for both the 20,000 and $50,000 \mu \mathrm{g} \mathrm{L}^{-1}$ standard mixtures. The trapped and concentrated analytes were then eluted for 2 minutes each with $100 \%$ acetonitrile followed by $100 \%$ ethyl acetate and $100 \%$ acetonitrile for thorough cleaning.

The optimum condition for the mobile phase to elute the trapped analyte in the SPE column into the analytical column was investigated. Initially, the mobile phase for the left pump was programmed with MSA-acetonitrile (45:55) at isocratic for 2.5 minutes, followed by 7 minutes gradient elution to $75 \%$ acetonitrile and 6 minutes icocratic at $75 \%$ acetonitrile. The first compound is eluted at 6.6 minutes, and the last compound is eluted at 11.55 minutes. Based on these preliminary observations, the chromatography conditions were adjusted and optimised to produce the best separation for all compound, as shown in Table 1, and the compounds were well separated as shown in Figure 3. The peaks between 8 and 10.5 minutes were due to column switching. For comparison, the order of the elution for DMP, DEP, and BBP in this study is consistent with the previous study [8].

The initial valve positions were left 6-1 and right 6-1 (or 6-1, 6-1) when the sample was loaded into the large-volume loops. The first column switched at $0.1 \mathrm{~min}$ (valve positions: $1-2,1-2)$, resulting in the enrichment of the compound in the SPE column, while all of the highly polar impurities were simultaneously flushed out. The second column switching (valve positions: 6-1, 1-2) occurred at 3.5 minutes and bypassed the large-volume loop, resulting in the shortening of the route of the mobile phase. Simultaneously, the gradient elution in the SPE column enabled the partial separation of the compounds. During the first and the second column switching, the analytical column was in the equilibrium mode. The third column switching (valve positions: 1-2, 6-1) occurred at $6.5 \mathrm{~min}$ and positioned the SPE column into the analytical flow path for eluting the bound analytes. Simultaneously, the large-volume loop was flushed with ethyl acetate for cleaning. The fourth column switching (valve positions: 6-1, 6-1) resulted in the manual loading of the sample onto the large-volume loop using a syringe under negative pressure, while the SPE and analytical columns were flushed with ethyl acetate for cleaning; both columns were left under equilibrium state.

3.2. Sample Pretreatment. The efficiency of the online SPEHPLC method was tested using spiked oil samples. Preliminary study showed that the online SPE-HPLC was able to give good separation by direct injection of oil without any sample pretreatment when the concentration of spike was in the range of 100 to $300 \mathrm{mg} \mathrm{L}^{-1}$. However, for the analysis of trace amounts of phthalates in the oil, interference from the bulk lipids was inevitable. Therefore, the removal of the fat is required, and a simple fat removal method was proposed.

In this study, the oil was diluted with n-hexane prior to the addition of the fat retainer (sorbent). The introduction of n-hexane was to reduce the viscosity of the oil and thus improve the surface interactions of the oil with the sorbent. The $\mathrm{n}$-hexane was later removed under a stream of nitrogen at $45^{\circ} \mathrm{C}$. The amount of $\mathrm{n}$-hexane has significant impact on the extraction efficiency. Insufficient amount of n-hexane resulted in highly viscous oil and hence resulted in less efficient surface interaction between the oil and the fat retainer, while excessive amount of $n$-hexane resulted in longer time needed for its complete removal from the mixture. The presence of $n$-hexane will affect the efficiency of the extractant (methanol:acetonitrile at 1:1) as the plasticizers have better affinity with $n$-hexane compared to the extractant. Different amounts of $\mathrm{n}$-hexane were tested and it was found that the optimal amount of $n$-hexane was $0.8 \mathrm{~mL}$ per $\mathrm{mL}$ of oil, with good recovery and acceptable relative 


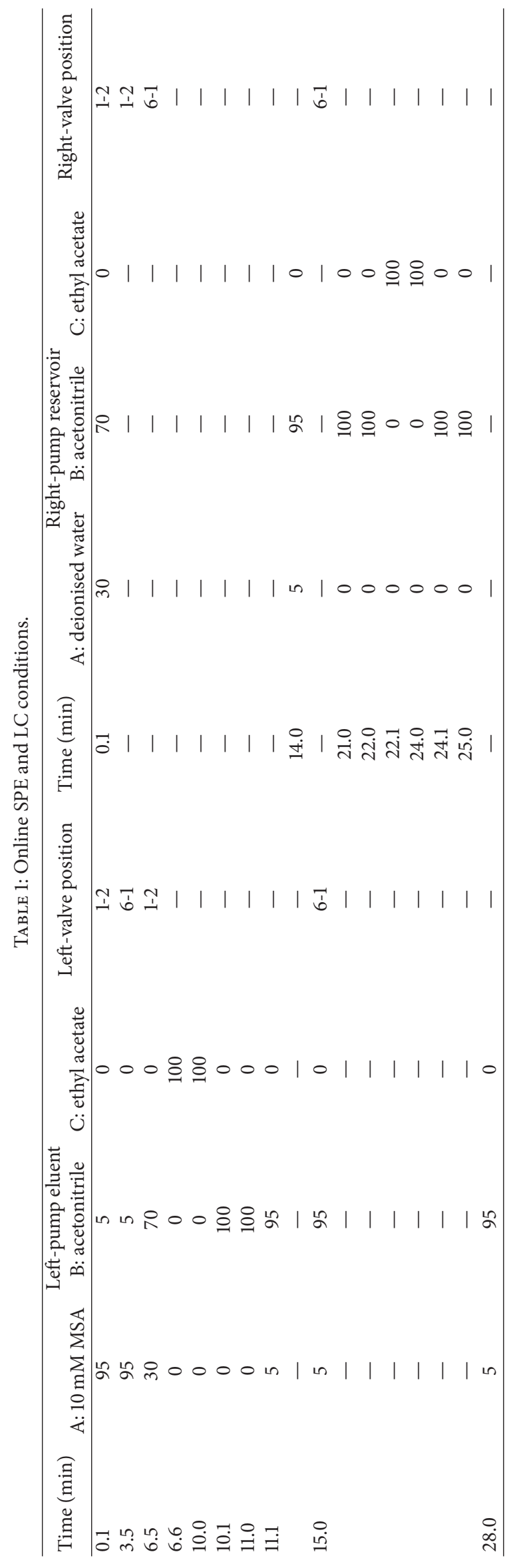




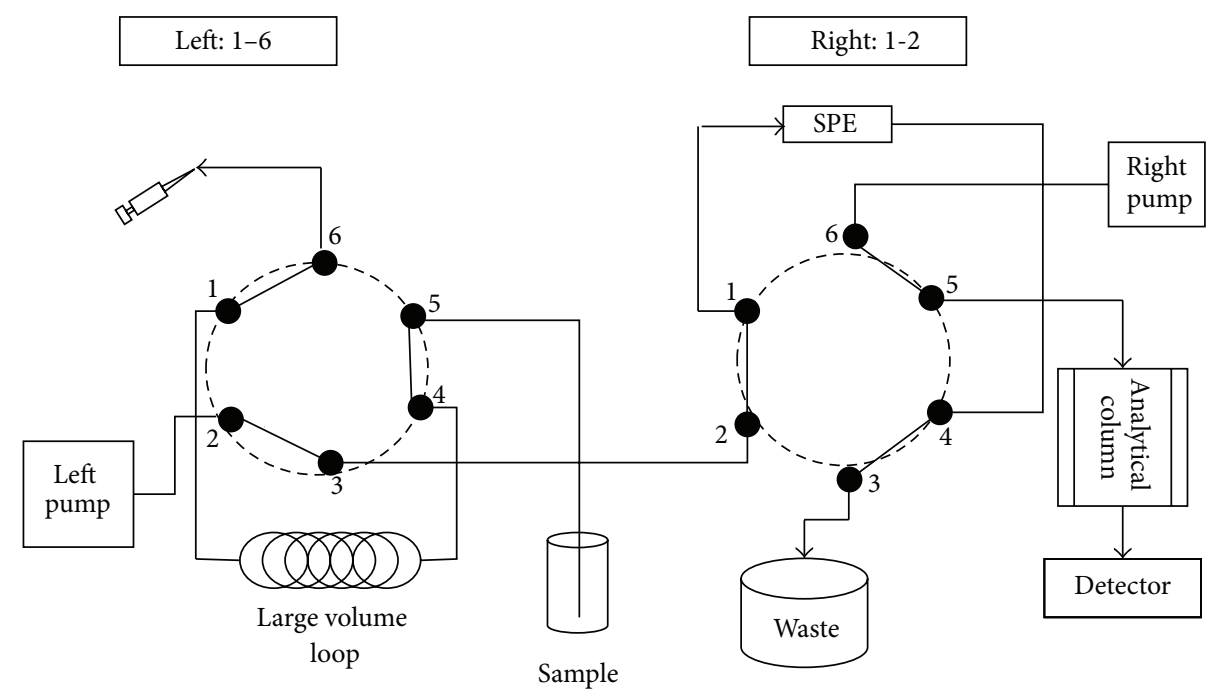

FIGURE 1: Left- and right-valve positions with the ports (1-6)

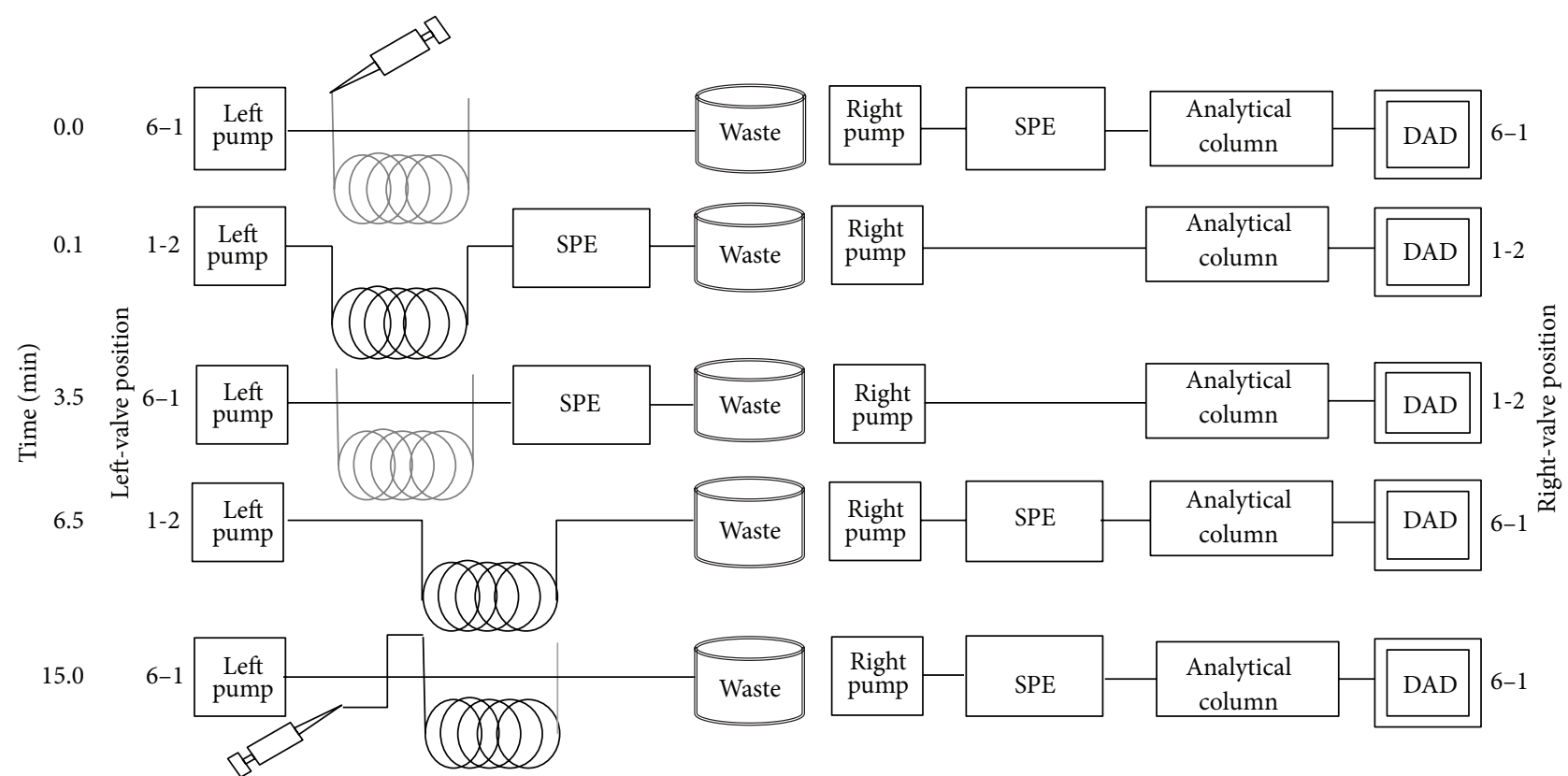

FIGURE 2: Schematic diagram of column switching during loading and enrichment phases.

standard deviations (RSD) being obtained using a spiked sample $\left(5 \mathrm{mg} \mathrm{L}^{-1}\right)$, as shown in Table 2 .

The application of fat retainers to eliminate fat was reported in the previous study [26]. In the analysis of PCBs in fat, different types of fat retainers (Florisil, basic alumina, neutral alumina, acidic alumina, and sulphuric acid impregnated silica) were used and different ratio of fat to fat retainer was tested. The extraction method used was accelerated solvent extraction (ASE) technique and it was found that the effective fat:fat retainer ratio was $1: 40$ for all fat retainers [27]. A study on contamination of phthalate esters, bisphenol A, bisphenol A diglycidyl ether, and nonylphenol in commercial whole milk using alumina, Extrelut, and Florisil as cleanup sorbents showed that the latter produced clean extracts [28]. In another study on the analysis of phthalates from ham using liquid-liquid extraction, the ratio of fat to fat retainer used was $1: 1.5$ [17]. In this study, the ratio of $1: 2.5$ was used to evaluate the efficiency of three different fat retainers: basic alumina, alumina active 90 neutral, and Florisil. Basic alumina produced good recovery and RSD, as shown in Table 3.

The extraction of the phthalates from the mixture of the palm oil and the fat retainer (basic alumina) is another challenge. Most of the common solvents that can dissolve these phthalates are also miscible with palm oil. The choice of solvents for the liquid-liquid extraction is thus limited. 


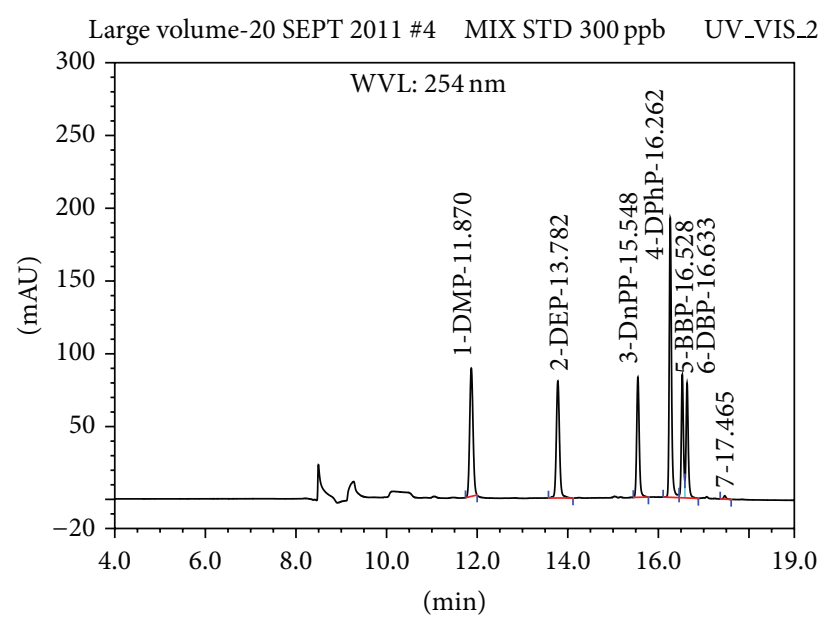

FIGURE 3: The chromatogram of six phthalate plasticizers.

TABLE 2: The effect of amount of $\mathrm{n}$-hexane ( $\mathrm{mL}$ per $\mathrm{mL}$ of oil), on the recovery and RSD of phthalates in samples spiked with $5 \mathrm{mg} \mathrm{L}^{-1}$.

\begin{tabular}{|c|c|c|c|c|c|c|c|c|}
\hline \multirow{3}{*}{$\begin{array}{l}\text { Compounds } \\
n=3\end{array}$} & \multicolumn{8}{|c|}{ Amount of n-hexane (mL) } \\
\hline & 1 & & 0.8 & & 0.5 & & 0 & \\
\hline & \% Recovery & $\%$ RSD & \% Recovery & $\%$ RSD & $\%$ Recovery & $\%$ RSD & $\%$ Recovery & $\%$ RSD \\
\hline DMP & 96.8 & 5.4 & 97.8 & 1.0 & 76.7 & 2.3 & 81.3 & 2.1 \\
\hline DEP & 81.4 & 5.7 & 72.8 & 5.3 & 36.1 & 5.8 & 69.6 & 3.3 \\
\hline DnPP & 74.0 & 6.3 & 89.7 & 3.1 & 63.7 & 4.0 & 72.2 & 5.5 \\
\hline $\mathrm{DPhP}$ & 73.5 & 0.9 & 89.9 & 2.2 & 67.8 & 0.8 & 69.6 & 9.0 \\
\hline BBP & 102.9 & 4.4 & 100.1 & 2.5 & 94.4 & 3.3 & 96.3 & 8.5 \\
\hline DBP & 101.7 & 1.0 & 100.7 & 2.7 & 73.6 & 3.4 & 86.7 & 4.9 \\
\hline
\end{tabular}

The previous study used acetonitrile to extract an antioxidant (Irganox 245) and an optical brightening agent (Uvitex OB) from olive oil [15]. The evaluation on two different solvents, for example, acetonitrile and methanol, was carried out on the analysis of the mixture of bis (2-ethylhexyl) phthalate (DEHP) and other plastic additives in olive oil [29]. The results showed that both solvents gave the same yield for the recovery of the additives, while the acetonitrile extract was clearer of interfering compound from the olive oil. A study on the migration on diphenylbutadiene (DPBD) from plastic to chocolate, chocolate spread, and margarine involves extraction of the fatty compound using n-hexane followed by liquid-liquid extraction using acetonitrile, with acceptable recovery [30]. In this study, the efficiency of two solvents, methanol and a mixture of methanol:acetonitrile at $1: 1$, was compared. It was found that the methanol: acetonitrile mixture (1:1) yielded better recovery, as shown in Table 4. The reason may be attributed to the ability of the mixture to extract a wider range of polarity of the phthalates from the palm oil.

In this online SPE-reverse phase HPLC system, both the SPE and the analytical column are nonpolar and the mobile phase must be polar. The sample in the large volume column, with the volume of approximately $3.5 \mathrm{~mL}$, will be passing through the C16 column for enrichment, similar to the process carried out in the usual offline SPE. The solvents used must be polar so that the phthalate plasticizers which are less and slightly polar can be trapped in the C16 column. This was achieved by the application of $5 \% 10 \mathrm{mM}$ of MSA in water during the preconcentration step.

As the extraction process was carried out using methanol:acetonitrile $(1: 1)$ and the sample introduction from the large loop into the SPE column must be in aqueous form, a solvent exchange step is required. However, the extractant must not be reduced to complete dry as some of the plasticizers like DBP are less soluble in water. A preliminary study was conducted to determine the maximum tolerable amount of the above extractant to give good recovery of the phthalates. A $10 \mathrm{ppm}$ standard was prepared in which a series of concentrations of the above extractant in water was injected into the system. It was found that the recovery of the standard is acceptable when the amount of methanol did not exceed 30\%. In this study, by reducing the amount of extractant to approximately $1 \mathrm{~mL}$ and subsequently making up to volume $(10 \mathrm{~mL})$ with water, which is about $10 \%$ of the extractant, the phthalates 
TABLE 3: Effect of the fat retainers on the recovery and repeatability of phthalate extraction from spiked samples $(1 \mathrm{mg} / \mathrm{L})$.

\begin{tabular}{|c|c|c|c|c|c|c|}
\hline \multirow{2}{*}{$\begin{array}{l}\text { Compounds } \\
n=3\end{array}$} & \multicolumn{2}{|c|}{$\begin{array}{l}\text { Aluminium oxide } \\
\text { (basic alumina) }\end{array}$} & \multicolumn{2}{|c|}{$\begin{array}{c}\text { Alumina active } 90 \\
\text { neutral }\end{array}$} & \multicolumn{2}{|c|}{ Florisil } \\
\hline & \% Recovery & $\%$ RSD & \% Recovery & $\%$ RSD & \% Recovery & $\%$ RSD \\
\hline DMP & 97.8 & 2.8 & 91.8 & 9.6 & 15.6 & 74.3 \\
\hline DEP & 59.4 & 2.3 & 78.1 & 8.8 & 29.1 & 20.8 \\
\hline DnPP & 103.8 & 0.9 & 0.0 & - & 0.0 & - \\
\hline $\mathrm{DPhP}$ & 93.3 & 3.6 & 70.6 & 25.3 & 0.0 & - \\
\hline $\mathrm{BBP}$ & 102.1 & 0.8 & 90.3 & 27.6 & 39.7 & 44.4 \\
\hline DBP & 94.0 & 3.3 & 74.1 & 31.8 & 23.3 & 44.6 \\
\hline
\end{tabular}

TABLE 4: Effect of the extraction solvent on phthalate recovery.

\begin{tabular}{lcc}
\hline Compounds & \multicolumn{2}{c}{$\begin{array}{c}\text { \% Recovery } \\
\text { MeOH : MeCN }(1: 1)\end{array}$} \\
\hline DMP & 60.0 & 89.9 \\
DEP & 83.9 & 73.7 \\
DnPP & 61.9 & 92.4 \\
DPhP & 49.2 & 83.8 \\
BBP & 56.3 & 96.8 \\
DBP & 72.7 & 84.5 \\
\hline
\end{tabular}

can successfully be trapped in the SPE column during the enrichment process. However, this solvent exchange step resulted in the dilution of the sample down to ten manifolds.

3.3. Method Optimization. The instrument was set up using the optimised conditions for two major steps; the first was for the enrichment and impurity washing in the SPE column and the second was for the separation, identification, and quantification of the phthalates in the analytical column. The optimization process also has to take into account the thorough columns washing with ethyl acetate and the sufficient time for columns equilibrium.

A calibration curve was constructed using the external standard method based on the area count, using Chromeleon v.6 software (Dionex, Sunnyvale, CA, USA). The calibration curve was linear in the range of $5-1000 \mu \mathrm{g} \mathrm{L}^{-1}$ with a correlation coefficient of 0.99 . The instrumental limit of detection (LODs) was $3 \mu \mathrm{g} \mathrm{L}^{-1}$ and limit of quantification (LOQs) was $10 \mu \mathrm{g} \mathrm{L}^{-1}$, which were calculated based on $3: 1$ and $10: 1$ signal to noise ratios, respectively [31].

3.4. Analysis of Cooking Oil. Palm oil spiked with $1 \mathrm{mg} \mathrm{L}^{-1}$ and $5 \mathrm{mg} \mathrm{L}^{-1}$ of standard was analysed as discussed above and the results are shown in Table 5. All phthalates produce good recovery for both concentrations, except for DEP which shows low recovery. A random analysis on a few samples of palm oil in the retail market was carried out on different brands of palm oil. Each of the palm oils was packed in poly ethylene terephthalate (PET) bottle. The result showed that only two phthalates were positively identified in the samples which were DBP and DMP as shown in Table 6. Sample A contained both phthalates while samples B and C contained
TABLE 5: The recoveries for the spiked samples.

\begin{tabular}{lcccc}
\hline \multirow{2}{*}{ Compounds } & \multicolumn{4}{c}{ Concentration of spiked samples (mg/L), $n=3$} \\
& \% Recovery & \% RSD & \% Recovery & \% RSD \\
\hline DMP & 97.8 & 2.8 & 97.8 & 1.0 \\
DEP & 59.4 & 2.3 & 101.4 & 1.3 \\
DnPP & 103.8 & 0.9 & 89.7 & 3.1 \\
DPhP & 93.3 & 3.6 & 89.9 & 2.2 \\
BBP & 102.1 & 0.8 & 100.1 & 2.5 \\
DBP & 94.0 & 3.3 & 100.7 & 2.7 \\
\hline
\end{tabular}

TABLE 6: The amount of phthalates plasticiser in different palm oil brands.

\begin{tabular}{lccc}
\hline & \multicolumn{3}{c}{ Amount of phthalates plasticiser $\left(\mathrm{mgL}^{-1}\right)$} \\
$\begin{array}{l}\text { Compounds } \\
n=3\end{array}$ & A & B & C \\
\hline DMP & n.d & n.d & n.d \\
DEP & n.d & n.d & n.d \\
DnPP & n.d & n.d & n.d \\
DPhP & n.d & n.d & n.d \\
BBP & $0.19 \pm 0.004$ & $0.91 \pm 0.05$ & n.d \\
DBP & $0.63 \pm 0.01$ & n.d. & $0.14 \pm 0.01$ \\
\hline
\end{tabular}

${ }^{*}$ N.d: not detected.

only BBP and DBP, respectively. The concentrations of all the phthalates were less than $1 \mathrm{mg} \mathrm{L}^{-1}$.

\section{Conclusion}

Online SPE-LC could be used to determine the amount of phthalate plasticisers in oil and required only minimal sample preparation steps to remove fat using $2.5 \mathrm{~g}$ of basic alumina and $0.8 \mathrm{~mL}$ of $\mathrm{n}$-hexane. Online SPE has the advantages of which the SPE column can be washed and reused, thanks to the high-pressure pump. Moreover, a cycle of SPE column enrichment, impurity removal, and column equilibrium can be achieved consistently. In the future, a thorough investigation on the contamination of the phthalates in palm oil in the retail market should be carried out on a wider spectrum of brands and to include various types of packaging material. 


\section{Conflict of Interests}

The authors declare that there is no conflict of interests regarding the publication of this paper.

\section{Acknowledgments}

The authors would like to acknowledge the financial supporter of this project which is the Ministry of Higher Education (MOHE), Malaysia (Project no. 600RMI/ST/FRGS/5.3.Fst/6/2010), and Universiti Teknologi MARA.

\section{References}

[1] J. Muncke, "Exposure to endocrine disrupting compounds via the food chain: is packaging a relevant source?" Science of the Total Environment, vol. 407, no. 16, pp. 4549-4559, 2009.

[2] K. Marsh and B. Bugusu, "Food packaging: roles, materials, and environmental issues: scientific status summary," Journal of Food Science, vol. 72, no. 3, pp. R39-R55, 2007.

[3] O.-W. Lau and S.-K. Wong, "Contamination in food from packaging material," Journal of Chromatography A, vol. 882, no. 1-2, pp. 255-270, 2000.

[4] M. Rahman and C. S. Brazel, "The plasticizer market: an assessment of traditional plasticizers and research trends to meet new challenges," Progress in Polymer Science, vol. 29, no. 12, pp. 1223-1248, 2004.

[5] E. Fasano, F. Bono-Blay, T. Cirillo, P. Montuori, and S. Lacorte, "Migration of phthalates, alkylphenols, bisphenol A and di(2ethylhexyl)adipate from food packaging," Food Control, vol. 27, no. 1, pp. 132-138, 2012.

[6] S. Biedermann-Brem, M. Biedermann, S. Pfenninger et al., "Plasticizers in PVC toys and childcare products: what succeeds the phthalates? Market survey 2007," Chromatographia, vol. 68, no. 3-4, pp. 227-234, 2008.

[7] M. A. Mohamed and A. S. Ammar, "Quantitative analysis of phthalates plasticizers in traditional Egyptian foods (Koushary and Foul Medams), black tea, instant coffee and bottled waters by solid phase extraction-capillary chromatography-mass spectroscopy," The American Journal of Food Technology, vol. 3, no. 5, pp. 341-346, 2008.

[8] K. Luks-Betlej, P. Popp, B. Janoszka, and H. Paschke, "Solidphase microextraction of phthalates from water," Journal of Chromatography A, vol. 938, no. 1-2, pp. 93-101, 2001.

[9] G. Latini, "Monitoring phthalate exposure in humans," Clinica Chimica Acta, vol. 361, no. 1-2, pp. 20-29, 2005.

[10] J. H. Petersent and T. Breindahl, "Plasticizers in total diet samples, baby food and infant formulae," Food Additives and Contaminants, vol. 17, no. 2, pp. 133-141, 2000.

[11] M. Wagner and J. Oehlmann, "Endocrine disruptors in bottled mineral water: total estrogenic burden and migration from plastic bottles," Environmental Science and Pollution Research, vol. 16, no. 3, pp. 278-286, 2009.

[12] T. Fierens, K. Servaes, M. van Holderbeke et al., "Analysis of phthalates in food products and packaging materials sold on the Belgian market," Food and Chemical Toxicology, vol. 50, no. 7, pp. 2575-2583, 2012.

[13] "Regulation (EC) No 1935/2004 of the European Parliament and of the Council of 27 October 2004 on materials and articles intended to come into contact with food and repealing Directives 80/590/EEC and 89/109/EEC," Official Journal of the European Union, vol. L338, pp. 4-16, 2004.

[14] A. S. Silva, R. S. García, I. Cooper, R. Franz, and P. P. Losada, "Compilation of analytical methods and guidelines for the determination of selected model migrants from plastic packaging," Trends in Food Science and Technology, vol. 17, no. 10, pp. 535-546, 2006.

[15] E. J. Quinto-Fernández, C. Pérez-Lamela, and J. Simal-Gándara, "Analytical methods for food-contact materials additives in olive oil simulant at sub-mg kg-1 level," Food Additives and Contaminants, vol. 20, no. 7, pp. 678-683, 2003.

[16] K. Holadová, G. Prokůpková, J. Hajšlová, and J. Poustka, "Headspace solid-phase microextraction of phthalic acid esters from vegetable oil employing solvent based matrix modification," Analytica Chimica Acta, vol. 582, no. 1, pp. 24-33, 2007.

[17] Z. Guo, S. Wang, D. Wei et al., "Development and application of a method for analysis of phthalates in ham sausages by solidphase extraction and gas chromatography-mass spectrometry," Meat Science, vol. 84, no. 3, pp. 484-490, 2010.

[18] J. D. Carrillo, M. P. Martínez, and M. T. Tena, "Determination of phthalates in wine by headspace solid-phase microextraction followed by gas chromatography-mass spectrometry. Use of deuterated phthalates as internal standards," Journal of Chromatography A, vol. 1181, no. 1-2, pp. 125-130, 2008.

[19] X. Li, Z. Zeng, Y. Chen, and Y. Xu, "Determination of phthalate acid esters plasticizers in plastic by ultrasonic solvent extraction combined with solid-phase microextraction using calix[4] arene fiber," Talanta, vol. 63, no. 4, pp. 1013-1019, 2004.

[20] J.-F. Jen and T.-C. Liu, "Determination of phthalate esters from food-contacted materials by on-line microdialysis and liquid chromatography," Journal of Chromatography A, vol. 1130, no. 1, pp. 28-33, 2006.

[21] Dionex, "Determination of polycyclic aromatic hydrocarbons (PAHs) in tap water using on-line solid-phase extraction followed by HPLC with UV and Fluorescence Detections," Application Notes 213, LPN 2128, 2009.

[22] Dionex, "Sensitive On-Line SPE-HPLC Determination of Paraquat and Diquat in Drinking and Environmental Waters," Application Notes 274, LPN 2726, 2011.

[23] Dionex, "Determination of vitamin B12 in beverages using online SPE followed by HPLC with UV detection," Application Notes 256, LPN 2574, 2010.

[24] Infocomm Commoditty Profile (Palm Oil), T. UNCTAD, Geneva, Switzerland, 2013.

[25] Z. Chen, P. Pavelic, P. Dillon, and R. Naidu, "Determination of caffeine as a tracer of sewage effluent in natural waters by on-line solid-phase extraction and liquid chromatography with diodearray detection," Water Research, vol. 36, no. 19, pp. 4830-4838, 2002.

[26] M. Järemo, E. Björklund, N. Nilsson, L. Karlsson, and L. Mathiasson, "Utilization of fat retainers in supercritical fluid extraction for the selective extraction of polychlorinated biphenyls from a model fat sample," Journal of Chromatography A, vol. 877, no. 1-2, pp. 167-180, 2000.

[27] E. Björklund, A. Muller, and C. von Holst, "Comparison of fat retainers in accelerated solvent extraction for the selective extraction of PCBs from fat-containing samples," Analytical Chemistry, vol. 73, no. 16, pp. 4050-4053, 2001.

[28] N. Casajuana and S. Lacorte, "New methodology for the determination of phthalate esters, bisphenol A, bisphenol A 
diglycidyl ether, and nonylphenol in commercial whole milk samples," Journal of Agricultural and Food Chemistry, vol. 52, no. 12, pp. 3702-3707, 2004.

[29] C. Simoneau and P. Hannaert, "Stability testing of selected plastics additives for food contact in EU aqueous, fatty and alternative simulants," Food Additives and Contaminants, vol. 16, no. 5, pp. 197-206, 1999.

[30] A. S. Silva, J. M. C. Freire, R. S. García, R. Franz, and P. P. Losada, "Time-temperature study of the kinetics of migration of DPBD from plastics into chocolate, chocolate spread and margarine," Food Research International, vol. 40, no. 6, pp. 679-686, 2007.

[31] M. M. Sanagi, S. L. Ling, Z. Nasir, D. Hermawan, W. A. Wan Ibrahim, and A. A. Naim, "Comparison of signal-to-noise, blank determination, and linear regression methods for the estimation of detection and quantification limits for volatile organic compounds by gas chromatography," Journal of AOAC International, vol. 92, no. 6, pp. 1833-1838, 2009. 

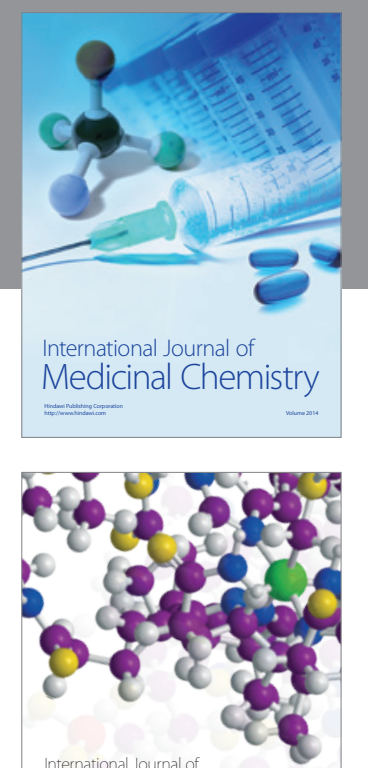

\section{Carbohydrate} Chemistry

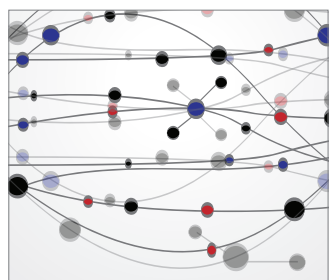

The Scientific World Journal
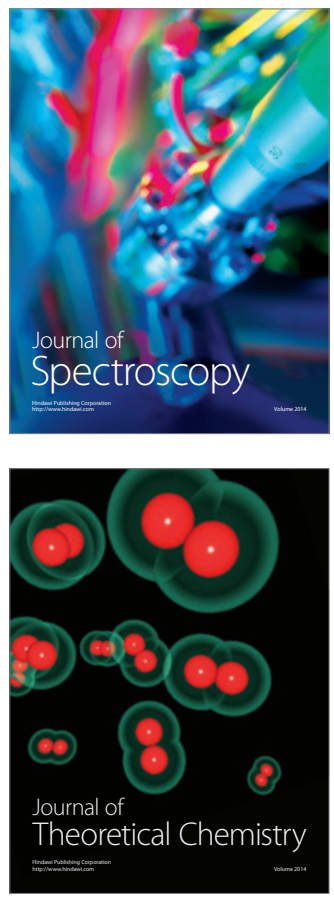
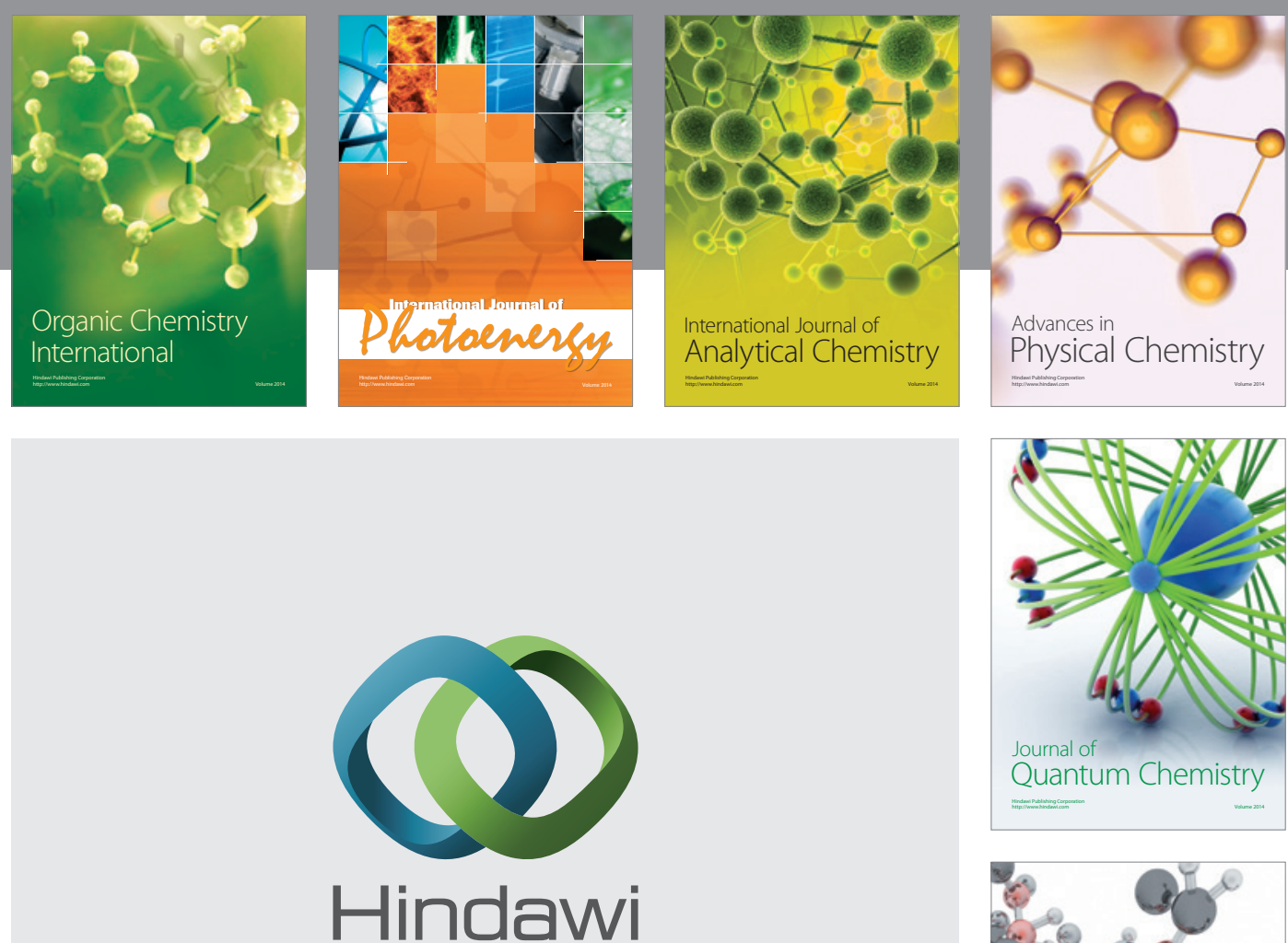

Submit your manuscripts at

http://www.hindawi.com

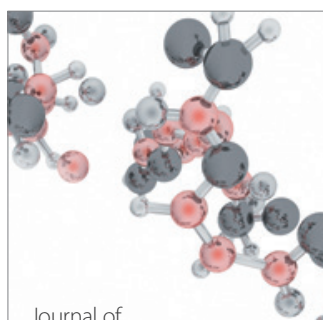

Analytical Methods

in Chemistry

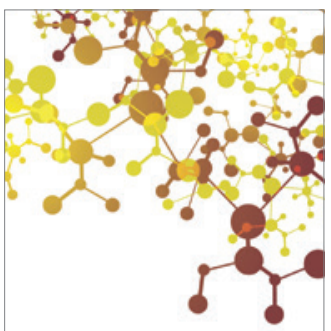

Journal of

Applied Chemistry

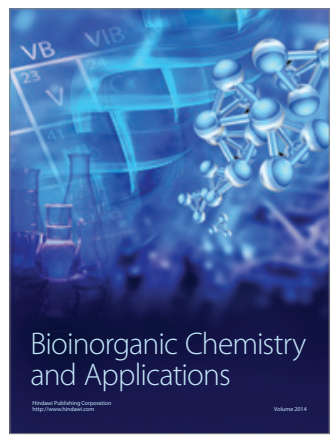

Inorganic Chemistry
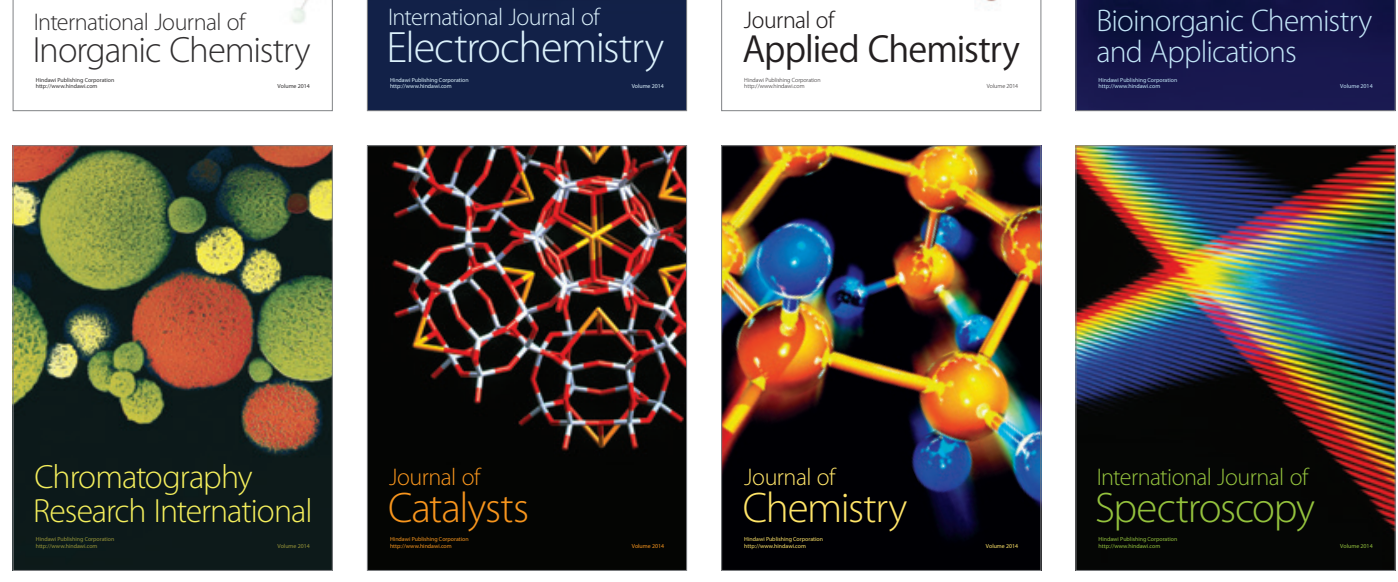